accepted for publication in AJ

\title{
The Nebular Environment and Enigmatic Hard X-ray Emission of the Hot DO White Dwarf KPD 0005+5106
}

\author{
You-Hua Chu ${ }^{1,2}$, and Robert A. Gruendl ${ }^{1}$, Rosa M. Williams ${ }^{1,2}$, \\ Theodore R. Gull ${ }^{2,3}$, Klaus Werner ${ }^{4}$ \\ chu@astro.uiuc.edu, gruendl@astro.uiuc.edu, rosanina@astro.uiuc.edu, \\ gull@sea.gsfc.nasa.gov, werner@astro.uni-tuebingen.de
}

\begin{abstract}
We have detected an ionized nebula around the hot DO white dwarf $\mathrm{KPD} 0005+5106$, and used the $[\mathrm{O} \mathrm{III}] / \mathrm{H} \alpha$ ratios and nebular velocities to separate this nebula from the background $\mathrm{H}$ II region of AO Cas. The angular size of the [O III] nebula of KPD $0005+5106$ is $\sim 3^{\circ}$. The velocity of this nebula is similar to those of the local interstellar $\mathrm{H}$ I gas and the interstellar/circumstellar absorption lines in UV spectra of KPD 0005+5106, but has a large offset from those of the stellar photospheric lines. The mass of the ionized nebula, $\sim 70 \mathrm{M}_{\odot}$, indicates that it consists of interstellar material and that the velocity offset between the star and the ambient medium should not be interpreted as a wind outflow. We have also analyzed the ROSAT PSPC observation of KPD 0005+5106 to determine its hard X-ray luminosity. Using the $L_{\mathrm{X}} / L_{\mathrm{bol}}$ relation for late-type stars and the lack of obvious near-IR excess of KPD 0005+5106, we exclude the possible existence of a binary companion with coronal activity. Without a wind outflow, the presence of stellar O VIII emission requires that X-rays at energies greater than $0.871 \mathrm{keV}$ are present in the vicinity of KPD $0005+5106$. This hard X-ray
\end{abstract}

\footnotetext{
${ }^{1}$ Department of Astronomy, University of Illinois at Urbana-Champaign, 1002 West Green Street, Urbana, IL 61801

2 Visiting Astronomer, Kitt Peak National Observatory, National Optical Astronomy Observatories, operated by the Association of Universities for Research in Astronomy, Inc. (AURA) under a cooperative agreement with the National Science Foundation.

${ }^{3}$ Goddard Space Flight Center, NASA, Code 681, Greenbelt, MD 20771-5302

${ }^{4}$ Institut für Astronomie und Astrophysik, Universität Tübingen, Sand 1, D-72076 Tübingen, Germany
} 
emission is most puzzling as neither photospheric emission at such high energies nor a high-temperature corona is expected from current stellar atmospheric models KPD 0005+5106. X-ray observations with high angular resolution and sensitivity are needed to confirm the positional coincidence and to enable X-ray spectral analyses for constraining the physical origin of the hard X-ray emission from KPD $0005+5106$.

Subject headings: white dwarfs - stars: individual (KPD 0005+5106) - planetary nebulae: general - H II regions

\section{Introduction}

About $20 \%$ of white dwarfs (WDs) exhibit a deficiency or an absence of hydrogen on their surfaces, and their evolutionary paths into such states are not well understood. If planetary nebulae $(\mathrm{PNe})$ are found around different types of WDs, the nebular properties may shed light on the evolutionary history of the stars. Only hot WDs can photoionize their nebulae and make them shine in $\mathrm{H} \alpha$. Therefore, Reynolds (1987) searched for $\mathrm{H} \alpha$ emission around 5 hot WDs and 4 sdO stars using a Fabry-Perot spectrometer, but he found emission only in the vicinity of the DO WDs PG 0108+101 and PG 0109+111, which are within $\sim 1^{\circ}$ from each other. Similarly, Werner et al. (1997) carried out a CCD imaging search for PNe around 16 hot WDs, but they detected $\mathrm{H} \alpha$ nebulosity in the vicinity of only PG $0109+111$. It was uncertain whether this ionized gas belonged to the interstellar medium or an evolved PN. Among the non-detections, the hot DO star KPD 0005+5106 presents a puzzle because its far ultraviolet spectrum shows strong $\mathrm{H}_{2}$ absorption lines indicating the presence of dense interstellar or circumstellar gas (Kruk \& Werner 1996, 1998). While this puzzle is partially solved by the recent detection of an O VI emission nebula by Otte, Dixon, \& Sankrit (2004), there remain many other unusual properties of KPD 0005+5106.

KPD 0005+5106 has a stellar effective temperature of 120,000 K (Werner, Heber, \& Fleming 1994), which is hot enough to photoionize the O VI-emitting nebula. It was the first WD reported to possess a corona heated by a wind, as its X-ray spectrum below $0.5 \mathrm{keV}$ could be satisfactorily fitted only by emission models of He-rich plasma at temperatures of $2-3 \times 10^{5} \mathrm{~K}$ (Fleming, Werner, \& Barstow 1993). This ongoing wind outflow has been used to explain the detection of stellar O VIII emission and circumstellar N V absorption in the high-resolution UV spectra of KPD 0005+5106 taken with the HST GHRS (Werner et al. 1996) and IUE SWP (Sion et al. 1997). These explanations should be reconsidered in light of the recent discovery of hard X-ray emission from KPD 0005+5106 near $1 \mathrm{keV}$ (O'Dwyer et al. 2003). This hard $\mathrm{X}$-ray emission is able to photoionize $\mathrm{O}^{+7}$ (ionization potential = 
$871 \mathrm{eV}$ ) to produce the observed O VIII recombination line emission, but the origin of this hard X-ray emission is not clear. The hard X-ray spectrum of KPD 0005+5106 indicates a plasma temperature greater than $10^{6} \mathrm{~K}$, which is too high to be explained by the He-rich wind invoked by Fleming et al. (1993). Hard X-ray emission is frequently associated with WDs in binary systems and is attributed to the coronal activity of a late-type companion or the accretion of a companion's material onto the surface of a WD; however, KPD 0005+5106 does not show a near-IR excess and is not known to have a late-type companion (O'Dwyer et al. 2003).

To conduct a more sensitive search for a coronally active companion of KPD 0005+5106, we followed a similar approach to that used by Gruendl et al. (2001) to illustrate the existence of a late-type dMe companion to the central star of the Helix Nebula. KPD 0005+5106 was monitored with high-dispersion spectra over four nights. No variations in stellar features were detected, but moderately bright nebular emission was detected throughout the entire slit centered on KPD 0005+5106. The nebular emission around KPD 0005+5106 consists of two velocity components. To determine the relationship between these nebular components and KPD 0005+5106, we have compared their velocities to those of photospheric and interstellar absorption lines in UV spectra of KPD 0005+5106, and examined images from four emissionline surveys. We find that one nebular component is photoionized by KPD 0005+5106 and the other component is associated with a background H II region. We have also analyzed a ROSAT pointed observation of KPD 0005+5106 to determine its hard X-ray luminosity and use it to constrain the possible existence of a late-type companion. In this paper, we describe the observations used in our investigation of KPD $0005+5106$ ( $\S 2$ ); analyze the nebular environment ( 33$)$, the nature of the ionized nebula ( $\$ 4)$, and hard X-ray emission (§5) of KPD $0005+5106$; and discuss the implications of our results (§6).

\section{Observations}

We have used four sets of wide-field emission-line images to examine the morphological and spectral properties of the ionized medium around KPD 0005+5106. We have also used high-dispersion optical echelle observations of KPD 0005+5106 to search for variability of spectral features and to study the nebular emission. Finally, archival ROSAT observations are used to quantitatively analyze the hard X-ray luminosity of KPD $0005+5106$. 


\subsection{Narrow-Band Wide-Field Images}

Four sets of images of the field of KPD $0005+5106$ are available. The first set of images are from an emission-line survey of the northern sky made by T. R. Gull and Y.-H. Chu in 1977-1979 using a Nikon 135-mm-focal-length lens and a Carnegie image tube with an RCA C33063 two-stage intensifier. The camera was piggybacked on the No. 3 0.4-m telescope at Kitt Peak National Observatory (KPNO). The instrumental setup was similar to that used by Parker, Gull, \& Kirshner (1979), but the field-of-view was larger, $\sim 16^{\circ}$, and the resulting plate scale was $26.5 \mathrm{~mm}^{-1}$. KPD $0005+5106$, at $l=116.1$ and $b=-10.9$, was well positioned in the survey field centered at $l=115^{\circ}$ and $b=-10^{\circ}$. Images were taken with $\mathrm{H} \alpha$ and [O III] filters for $720 \mathrm{~s}$ and $900 \mathrm{~s}$, respectively, and recorded on hyper-sensitized Kodak IIIa-J photographic plates. The $\mathrm{H} \alpha$ filter, centered at $6570 \AA$ with a FWHM of 75 $\AA$, transmitted both the $\mathrm{H} \alpha$ and [N II] $\lambda \lambda 6548,6583$ lines. The [O III] filter, centered at $5010 \AA$ with a FWHM of $28 \AA$, transmitted only the [O III] $\lambda 5007$ line.

The second set of images of KPD 0005+5106 were taken by T. R. Gull in 1980 October as a follow-up study of interesting objects discovered from the Emission-Line Survey of the Milky Way (Parker et al. 1979). The instrumental setup differs from the one described above in that a Nikon 300-mm-focal-length objective lens was used and the field-of-view was 7.1. Images were taken with $\mathrm{H} \alpha$ and [O III] filters for $720 \mathrm{~s}$ and $1200 \mathrm{~s}$, respectively.

A third wide-field $\mathrm{H} \alpha$ image with higher sensitivity was available from the Virginia Tech Spectral-Line Survey ${ }^{1}$. The $\mathrm{H} \alpha$ image from this survey used a $17 \AA$ wide filter and thus rejected the flanking $\left[\mathrm{N}\right.$ II] lines. The field of view was $\sim 10^{\circ}$ in diameter, and the image scale was 1'6 pixel ${ }^{-1}$. The detection limit of the $\mathrm{H} \alpha$ image was $\gtrsim 2 \mathrm{pc} \mathrm{cm}^{-6}$ in emission measure.

A fourth wide-field view of the region around KPD 0005+5106 was obtained from the Wisconsin H-Alpha Mapper Northern Sky Survey (WHAM-NSS) data release version 1.0 (Haffner et al. 2003). The WHAM-NSS data had a coarse spatial resolution, $\sim 1^{\circ}$, but recorded a high-resolution $\left(\sim 12 \mathrm{~km} \mathrm{~s}^{-1}\right)$ spectrum at each position. We retrieved WHAMNSS data for a large region centered at KPD 0005+5106. The data were re-gridded spatially onto a common Galactic coordinate system with $10^{\prime}$ pixels using a simple nearest-neighbor tiling algorithm. The data were also interpolated and re-gridded spectrally onto a common local standard of rest velocity frame, i.e., $V_{\mathrm{LSR}}$, from -100 to $+100 \mathrm{~km} \mathrm{~s}^{-1}$ with $5 \mathrm{~km} \mathrm{~s}^{-1}$ pixels. The final product is an image-velocity cube covering $28^{\circ} \times 28^{\circ} \times 200 \mathrm{~km} \mathrm{~s}^{-1}$.

\footnotetext{
${ }^{1}$ Available at http://www.phys.vt.edu/ halpha/.
} 


\subsection{High-Dispersion Echelle Spectroscopy}

We obtained high-dispersion spectroscopic observations of KPD 0005+5106 with the echelle spectrograph on the $4 \mathrm{~m}$ telescope at KPNO on 2003 November 4-7. These observations were made with a 79 line $\mathrm{mm}^{-1}$ echelle grating, a 226 line $\mathrm{mm}^{-1}$ cross-disperser and a broad-band blocking filter (GG385). The long-focus red camera was used to obtain a reciprocal dispersion of $3.5 \AA \mathrm{mm}^{-1}$ at $\mathrm{H} \alpha$. The spectra were imaged using the T2KB CCD, of which the $24 \mu \mathrm{m}$ pixel size corresponded to $0^{\prime \prime} .26$ pixel $^{-1}$ along the slit and $\sim 0.08 \AA$ pixel $^{-1}$ along the dispersion axis. A $2^{\prime \prime}$ slitwidth was used, resulting in an instrumental resolution of $0.3 \AA\left(14 \mathrm{~km} \mathrm{~s}^{-1}\right.$ at $\left.\mathrm{H} \alpha\right)$, as measured by the FWHM of the unresolved telluric emission lines. A slit length of $15^{\prime \prime}$ was used for these multi-order observations so that a wavelength coverage of 4500-7500 $\AA$ was achieved. The slit was north-south oriented and centered on KPD $0005+5106$.

Each night the echelle observation of KPD 0005+5106 was split into two 900 s exposures to aid in cosmic-ray removal. The observations were reduced using standard packages in $\mathrm{IRAF}^{2}$. All spectra were bias and dark corrected, and cosmic ray events were rejected. Observations of a Th-Ar lamp were used for wavelength calibration, and the telluric lines in the source observations were used for a precise velocity alignment. The accuracy of velocity measurements is $\pm 2 \mathrm{~km} \mathrm{~s}^{-1}$ in the $\mathrm{H} \alpha+[\mathrm{N} \mathrm{II}]$ order and $\pm 5 \mathrm{~km} \mathrm{~s}^{-1}$ in the [O III] order. Absolute flux calibration was not possible due to the full moon and variable clouds in the sky during the echelle observing run. Fortunately, absolute flux calibration is not needed for extracting velocity profiles from the echelle data.

Nebular $\mathrm{H} \alpha$, [O III] $\lambda 5007$, and [N II] $\lambda 6584$ lines are detected at levels of 2-4 counts pixel $^{-1}$ above a sky background of $4-5$ counts pixel ${ }^{-1}$. The velocity profiles of these three lines are extracted from regions $5^{\prime \prime}$ above and $5^{\prime \prime}$ below the spectrum of KPD 0005+5106 with the sky emission approximated as a constant and subtracted. No other nebular lines were detected in the echelle observations.

\subsection{ROSAT X-ray Observations}

KPD 0005+5106 was observed by the ROSAT Position Sensitive Proportional Counter (PSPC) with a boron filter on 1991 December 31 for 5 ks (Observation number RF200428;

\footnotetext{
${ }^{2}$ IRAF is distributed by the National Optical Astronomy Observatories, which are operated by the Association of Universities for Research in Astronomy, Inc., under cooperative agreement with the National Science Foundation.
} 
PI: M. Barstow). The PSPC is sensitive to photon energies between 0.1 and $2.4 \mathrm{keV}$. The boron filter, having little transmission between 0.188 and $0.28 \mathrm{keV}$, was designed to provide additional spectral information at this soft energy band, but it also reduced the transmission at $1 \mathrm{keV}$ by $\sim 20 \%$.

\section{Nebular Environment of KPD $0005+5106$}

We first noticed an ionized nebula around KPD 0005+5106 from our echelle observations, which revealed nebular line emission throughout the $15^{\prime \prime}$ slit length. Only the nebular $\mathrm{H} \alpha$, [N II] $\lambda 6583$, and [O III] $\lambda 5007$ lines are well detected; their extracted velocity profiles are shown in Figure 1. The $\mathrm{H} \alpha$ and [N II] line profiles are similar, barring their different thermal widths and the contaminating geocoronal $\mathrm{H} \alpha$ emission near $0 \mathrm{~km} \mathrm{~s}^{-1}$ observed velocity, or $V_{\mathrm{LSR}} \sim 3 \mathrm{~km} \mathrm{~s}^{-1}$; both lines show velocity components at $V_{\mathrm{LSR}} \sim-7$ and -32 $\mathrm{km} \mathrm{s}^{-1}$. The [O III] line, on the other hand, shows only one velocity component near -3 $\mathrm{km} \mathrm{s}^{-1}$, which is most likely associated with the $-7 \mathrm{~km} \mathrm{~s}^{-1}$ component of the $\mathrm{H} \alpha$ and [N II] lines. The drastically different $\left[\mathrm{O}\right.$ III] $/ \mathrm{H} \alpha$ ratios between the -7 and $-32 \mathrm{~km} \mathrm{~s}^{-1}$ velocity components indicate that these two components are associated with ionizing stars of very different effective temperatures.

The two sets of $\mathrm{H} \alpha$ and [O III] images taken with an image tube camera and a 135-mm or 300-mm objective lens are shown in Figures 2 and 3. Despite the different degrees of vignetting in the field-of-view, both sets of images show that the $\mathrm{H} \alpha$-emitting region is more extended than the [O III]-emitting region and that the $[\mathrm{O}$ III] region is roughly centered on KPD 0005+5106. A deeper $\mathrm{H} \alpha$ image is available from the Virginia Tech Emission-Line Survey $^{3}$, and it shows that KPD $0005+5106$ is superposed on the western part of an $\mathrm{H} \alpha$ emission region about $4^{\circ}$ across.

The iso-velocity maps made with the WHAM-NSS data provide a complete view of the emission region at a lower spatial resolution. As shown in Figure 4, two spatially distinct emission regions near KPD 0005+5106 can be identified with central velocities similar to the two velocity components revealed in the $\mathrm{H} \alpha$ and [N II] line profiles from our echelle observations. The emission region associated with the $-32 \mathrm{~km} \mathrm{~s}^{-1}$ component is brighter. It is $\sim 4^{\circ}$ across and best seen in the iso-velocity maps near $-30 \mathrm{~km} \mathrm{~s}^{-1}$; its brightest patch of emission can be traced from -5 to $-55 \mathrm{~km} \mathrm{~s}^{-1}$. This $-32 \mathrm{~km} \mathrm{~s}^{-1}$ emission region has been identified to be the H II region of AO Cas at a distance of $3.7 \mathrm{kpc}$ (Reynolds 1988). AO Cas is a binary system with the primary being an O9 III star and the secondary being of a similar

\footnotetext{
${ }^{3}$ Available at http://www.phys.vt.edu/ halpha/fields/Cas01/.
} 
spectral type (Walborn 1973; Gies \& Wiggs 1991). The absence of [O III] emission from this H II region (see Figs. 2b \& 3b) is consistent with the late-O spectral types of the AO Cas binary system. Therefore, this H II region is a background object to KPD $0005+5106$.

The emission region associated with the $-7 \mathrm{~km} \mathrm{~s}^{-1}$ component is faint, and the superposition of the emission from the $\mathrm{H}$ II region of $\mathrm{AO}$ Cas makes it difficult to identify the boundary of this $-7 \mathrm{~km} \mathrm{~s}^{-1}$ component. The spatial extent of the $-7 \mathrm{~km} \mathrm{~s}^{-1}$ emission region is better seen in the iso-velocity maps at $V_{\mathrm{LSR}} \geq-10 \mathrm{~km} \mathrm{~s}^{-1}$, where the contamination from the red wing of the $-32 \mathrm{~km} \mathrm{~s}^{-1}$ component is negligible. The brightest region of the -7 $\mathrm{km} \mathrm{s}^{-1}$ component is a $3^{\circ}$-long horizontal band of emission below KPD 0005+5106. On the two ends of this band of emission, fainter filaments of emission curve toward lower galactic latitudes and form an apparent shell structure about $10^{\circ}$ across. This $-7 \mathrm{~km} \mathrm{~s}^{-1} \mathrm{H} \alpha$ emission region is much more extended than the [O III] region shown in Figure 2b; only the brightest part of the $\mathrm{H} \alpha$ emission region overlaps with the [O III] region. It is not clear whether the apparent shell structure is associated with KPD $0005+5106$ or a result by chance superposition. As KPD 0005+5106 has an effective temperature of 120,000 K (Werner et al. 1994), its photoionized region should have high excitation with high $[\mathrm{O}$ III $] / \mathrm{H} \alpha$ ratios. Therefore, we suggest that only the [O III]-emitting region of the $-7 \mathrm{~km} \mathrm{~s}^{-1}$ component is associated with KPD 0005+5106.

\section{The Nature of the Nebula Photoionized by KPD 0005+5106}

Is the $-7 \mathrm{~km} \mathrm{~s}^{-1}$ ionized gas associated with KPD $0005+5106$ interstellar or circumstellar in origin? The UV spectra of KPD $0005+5106$ show photospheric absorption lines at an average $V_{\mathrm{LSR}}$ of $+42 \mathrm{~km} \mathrm{~s}^{-1}$ and interstellar/circumstellar absorption lines at an average $V_{\mathrm{LSR}}$ of $-7 \mathrm{~km} \mathrm{~s}^{-1}$ (converted from the $V_{\text {hel }}$ reported by Werner et al. 1996). It might be hypothesized that the interstellar/circumstellar absorption arises from the approaching side of an expanding PN of KPD 0005+5106. The systemic velocity of the PN should then be similar to the velocity of the central star, unless the PN has been braked by dense interstellar medium. Adopting the average photospheric absorption line velocity of KPD 0005+5106 as the systemic velocity of the PN, the expansion velocity of the PN would be nearly $50 \mathrm{~km}$ $\mathrm{s}^{-1}$ and we would expect to see $\mathrm{H} \alpha$ and [O III] emission from the receding side of the PN at $+90 \mathrm{~km} \mathrm{~s}^{-1}$. Our echelle observations do not show any nebular emission at this velocity; thus, we may rule out the expanding PN hypothesis.

It is likely that the ionized nebula of KPD $0005+5106$ consists of interstellar material. The interstellar $\mathrm{H} \mathrm{I}$ in this direction spans a wide range of velocities, but the main component is at roughly $-15 \leq V_{\mathrm{LSR}} \leq+5 \mathrm{~km} \mathrm{~s}^{-1}$ and has a smooth spatial distribution on scales of 
$5^{\circ}-10^{\circ}$ (Hartmann \& Burton 1997). The smooth morphology and velocity distribution of the interstellar H I gas are both similar to those of the nebula photoionized by KPD 0005+5106, supporting the interstellar origin of the nebula.

The size and mass of the nebula ionized by KPD 0005+5106 provide further information about its origin. The distance to KPD $0005+5106$ has been determined spectroscopically to be $270_{-120}^{+210}$ pc (Werner et al. 1994). The $3^{\circ}$ angular size of the [O III]-emitting region corresponds to a linear size of $14_{-6}^{+11} \mathrm{pc}$. This size rivals that of "the largest PN" recently discovered around PG 1034+001 by Hewett et al. (2003) and its enormous ionized halo subsequently reported by Rauch, Kerber, \& Pauli (2004). However, we find an interstellar origin to be more probable for the nebula of PG 1034+001 because its nebular velocity $(\sim 0$ $\mathrm{km} \mathrm{s}^{-1}$, Hewett et al. 2003) is similar to those of the low-velocity local interstellar H I gas (Hartmann \& Burton 1997) and the circumstellar/interstellar absorption lines $\left(+7 \mathrm{~km} \mathrm{~s}^{-1}\right.$, Holberg, Barstow, \& Sion 1998), but very different from those of the photospheric lines of PG 1034+001 (+51 $\mathrm{km} \mathrm{s}^{-1}$, Holberg et al. 1998). Confirmed PNe are all much smaller than the ionized nebula of KPD $0005+5106$.

To determine the mass of the ionized nebula of KPD 0005+5106, we use the WHAM-NSS $\mathrm{H} \alpha$ line integrated over a $2^{\circ}$-radius aperture centered on KPD $0005+5106$. The integrated $\mathrm{H} \alpha$ line profile is fitted and decomposed into two Gaussian components at -7 and $-32 \mathrm{~km} \mathrm{~s}^{-1}$. The $\mathrm{H} \alpha$ flux of the $-7 \mathrm{~km} \mathrm{~s}^{-1}$ component is used in the mass estimate. Adopting a distance of $270 \mathrm{pc}$, we find that the total $\mathrm{H} \alpha$ luminosity of the gas in the $-7 \mathrm{~km} \mathrm{~s}^{-1}$ component to be $\sim 2 \times 10^{34} \mathrm{ergs} \mathrm{s}^{-1}$. Assuming a homogeneous Strömgren sphere, we find an rms density of $\sim 0.8 \mathrm{H}$-atom $\mathrm{cm}^{-3}$ and the total mass of the ionized nebula of KPD $0005+5106$ to be 70 $\mathrm{M}_{\odot}$. This mass is clearly too large for a PN. Therefore, we conclude that the $-7 \mathrm{~km} \mathrm{~s}^{-1}$ nebula photoionized by KPD 0005+5106 consists of interstellar material.

As a consistency check, we have also evaluated the ionization requirement. The $\mathrm{H} \alpha$ luminosity of the ionized gas requires an ionizing luminosity of $1.4 \times 10^{46}$ photons s${ }^{-1}$. Synthetic stellar fluxes calculated for hot central stars of planetary nebulae are available at http://astro.uni-tuebingen.de/ rauch/. Using a helium NLTE model for $T_{\text {eff }}=120,000 \mathrm{~K}$ and $\log g=7$ (in cgs units) and adopting a stellar mass of $0.59 \mathrm{M}_{\odot}$ for KPD $0005+5106$

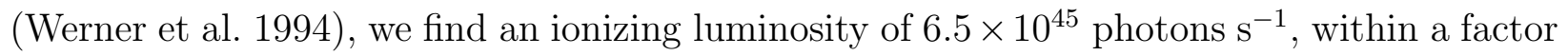
of 2 from that required to ionize the gas. The good agreement between the expected and required ionizing luminosities suggests that the spectroscopic distance of $270 \mathrm{pc}$ is reasonably accurate. 


\section{Nature of the Hard X-ray Emission from KPD 0005+5106}

The nature of the spatially-unresolved hard X-ray emission from KPD 0005+5106 is puzzling. Hard X-ray emission from WDs is usually attributed to their binary companions, and a late-type companion can be diagnosed by a near-IR excess; however, KPD 0005+5106 shows no obvious near-IR excess, with $V=13.32, J=13.93, H=14.13$, and $K=14.18$ (O'Dwyer et al. 2003). The absence of detectable near-IR excess places an upper limit on the luminosity and spectral type of a companion. The hard X-ray luminosity of KPD $0005+5106$ also constrains the luminosity of a hidden companion, as $L_{\mathrm{X}} / L_{\mathrm{bol}} \sim 10^{-3}-10^{-4}$ for coronal X-ray emission from late-type stars (Fleming, Schmitt, \& Giampapa 1995). Below we use the near-IR and X-ray constraints to assess the possibility of a hidden late-type companion that is responsible for the hard X-ray emission from KPD 0005+5106.

The ROSAT PSPC observation RF200428 detected a total of $25 \pm 5$ counts (backgroundsubtracted) from KPD $0005+5106$ in the $0.4-2.0 \mathrm{keV}$ band. To convert the count rate to luminosity, we use the energy-count conversion factors for ROSAT PSPC observations with a boron filter in the hard band $(\geq 0.4 \mathrm{keV})$ for thermal line spectra provided in Figure 10.12 of the ROSAT Mission Description. We adopt $N_{\mathrm{H}}=5 \times 10^{20} \mathrm{H}$-atoms $\mathrm{cm}^{-2}$ derived from Lyman $\alpha$ absorption profile by Werner et al. (1994) as the absorption column density. For plasma temperatures of $2-5 \times 10^{6} \mathrm{~K}$, the conversion factor is $0.4-3.5 \times 10^{10} \mathrm{counts}^{2}$ $\mathrm{erg}^{-1}$. The unabsorbed hard X-ray flux from KPD $0005+5106$ is thus $0.14-1.3 \times 10^{-12}$ ergs $\mathrm{cm}^{-2} \mathrm{~s}^{-1}$.

The full range of uncertainty in the spectroscopic distance to KPD 0005+5106, 270 -210 pc (Werner et al. 1994), is considered in the discussion below. Using the near distance (150 pc), the X-ray luminosity of KPD $0005+5106$ is $0.4-4 \times 10^{30} \mathrm{ergs} \mathrm{s}^{-1}$. If the hard $\mathrm{X}$-ray emission originates from the corona of a late-type companion, its $L_{\mathrm{bol}}$ is at least $4 \times 10^{32}-4 \times 10^{34} \mathrm{ergs} \mathrm{s}^{-1}$, corresponding to $M_{\mathrm{bol}}=7.2-2.2$. Assuming that the companion is a main sequence star, the least luminous possible spectral type is M0. An M0 V star at a distance of $150 \mathrm{pc}$ would have $K=11.1$, and would be too luminous for KPD 0005+5106's $K=14.18$. Using the far distance $(480 \mathrm{pc})$, the X-ray luminosity of KPD $0005+5106$ is $0.4-4 \times 10^{31} \mathrm{ergs} \mathrm{s}^{-1}$. Using the same derivation, we find the faintest possible spectral type for a coronal companion is G5. A G5V star at a distance of $480 \mathrm{pc}$ would have $K=11.7$, and would also be too luminous to be hidden by KPD 0005+5106. Therefore, the near-IR photometric measurements and the hard X-ray luminosity of KPD 0005+5106

exclude the possible existence of a late-type companion whose corona contributes to the observed hard X-ray emission. This conclusion is also supported by our echelle observations of KPD 0005+5106, which did not detect any $\mathrm{H} \alpha$ emission from a late-type companion.

The discovery of a photoionized nebula around KPD 0005+5106 opens up another possi- 
ble mechanism to generate X-ray emission: accretion of interstellar material onto the stellar surface. The rate of gravitational energy released by the accreted material is $\pi G R_{*} M_{*} v \rho_{0}$, where $G$ is the gravitational constant, $R_{*}$ is the stellar radius, $M_{*}$ is the stellar mass, $v$ is the translational velocity of star through the interstellar gas, and $\rho_{0}$ is the density of the ambient interstellar gas. Adopting $R_{*}=2.8 \times 10^{9} \mathrm{~cm}$ and $M_{*}=0.59 \mathrm{M}_{\odot}$ as determined from the spectral analysis of Werner et al. (1994), a translational velocity of $40 \mathrm{~km} \mathrm{~s}^{-1}$, and an interstellar density of $1 \mathrm{H}$-atom $\mathrm{cm}^{-3}$, we find an accretion luminosity $\sim 6 \times 10^{18}$ $\operatorname{ergs~} \mathrm{s}^{-1}$, more than twelve orders of magnitude lower than the hard X-ray luminosity of KPD 0005+5106. Therefore, we conclude that the accretion of interstellar material does not contribute much to the hard X-ray emission.

Is the hard X-ray emission from KPD 0005+5106 intrinsic or extrinsic? The angular resolution of the ROSAT PSPC, $\sim 30^{\prime \prime}$, is not able to resolve background sources projected within a few arcsec from KPD 0005+5106. However, the stellar O VIII emission from KPD 0005+5106 (Werner et al. 1996; Sion et al. 1997) can be used to argue for a local, instead of background, origin of the hard X-rays. The O VIII recombination line emission requires the ionization of $\mathrm{O}^{+7}$, which may be achieved through thermal collisions in wind outflows or photoionization by X-rays at energies greater than $0.871 \mathrm{keV}$. The suggestion of a wind outflow of KPD 0005+5106 was based on the velocity offset between the stellar photospheric lines and the "circumstellar" absorption lines (Sion \& Downes 1992); however, we have demonstrated in $\S 4$ that this "circumstellar" material is really interstellar medium. There is therefore no evidence for a wind outflow. Without a wind outflow, a hard $(>0.871$ $\mathrm{keV}$ ) X-ray radiation field is needed, and the source has to be local to KPD 0005+5106. We have ruled out the existence of a late-type companion with a corona; thus, KPD 0005+5106 must be responsible for the hard X-ray emission. Stellar X-ray emission may originate from the photosphere or a corona; however, X-ray emission near $1 \mathrm{keV}$ is not expected from models of the atmosphere of KPD 0005+5106 and WDs are not expected to possess hot coronae. The hard X-ray emission from KPD $0005+5106$ is most puzzling.

\section{Summary and Conclusions}

We have detected a large ionized nebula encompassing both KPD 0005+5106 and AO Cas. The nebular velocities and $[\mathrm{O}$ III $] / \mathrm{H} \alpha$ ratios are used to separate the high-excitation nebula ionized by KPD $0005+5106$ and the background H II region ionized by AO Cas. The nebula ionized by KPD 0005+5106 contains interstellar gas because its radial velocity is similar to that of the local interstellar medium and the nebular mass $\left(\sim 70 \mathrm{M}_{\odot}\right)$ is much higher than those of planetary nebulae. 
The interstellar origin of the nebula removes the basis for the suggestion of a wind outflow from KPD $0005+5106$ - an offset of about $+50 \mathrm{~km} \mathrm{~s}^{-1}$ between the nebular absorption line velocities and the photospheric line velocities. The recently discovered large ionized nebula around the hot DO WD PG 1034+001 (Hewett et al. 2003) also shows a nebular velocity similar to the circumstellar/interstellar absorption line velocities and the velocity of local interstellar gas but offset by about $+45 \mathrm{~km} \mathrm{~s}^{-1}$ from the stellar photospheric line velocities (Holberg et al. 1998). We suggest that the large ionized nebula around PG 1034+001 is also interstellar in origin. The large ionized nebulae of KPD 0005+5106 and PG 1034+001 clearly demonstrate that hot WDs contribute to the ionization of the interstellar medium. The brightest hot WDs are also the nearest, within a few hundred pc; thus, their photoionized interstellar nebulae would be angularly large so that the WHAM-NSS provides the best data set to search for these nebulae.

We have analyzed the hard X-ray luminosity of KPD $0005+5106$. Using the $L_{\mathrm{X}} / L_{\mathrm{bol}}$ relation for typical late-type stars with active coronae and KPD 0005+5106's lack of obvious near-IR excess, we have excluded the possibility that a late-type companion's coronal activity is responsible for the hard X-ray emission from KPD 0005+5106. The stellar O VIII emission of KPD $0005+5106$ places useful constraints on the location of the hard X-ray source. Our analysis of the nebular environment of KPD $0005+5106$ abrogates the previous suggestion of a wind outflow from KPD $0005+5106$; thus, a local hard X-ray radiation field is needed to photoionize $\mathrm{O}^{+7}$ in order to produce the stellar O VIII emission.

We conclude that the most likely origin of the hard X-ray emission from KPD 0005+5106 is the DO WD itself, either from its photosphere or a corona, although such photospheric emission is not expected from the currently available stellar atmospheric models and there are no known mechanisms to generate hot coronae around hot WDs. To further study the origin of hard X-ray emission from KPD $0005+5106$, X-ray observations with high angular resolution and high sensitivity are needed to confirm the positional coincidence between the hard X-ray source and KPD 0005+5106 and to obtain high-quality data for spectral analysis. Future observations with Chandra X-ray Observatory and XMM-Newton Observatory are most desirable.

This research has used data from the Wisconsin H-Alpha Mapper, which is funded by the National Science Foundation. YHC acknowledges the support of NASA grant NAG 5-13076. X-ray data analysis in Tübingen is supported by the DLR under grant 50 OR 0201. 


\section{REFERENCES}

Fleming, T. A., Schmitt, J. H. M. M., \& Giampapa, M. S. 1995, ApJ, 450, 401

Fleming, T. A., Werner, K., \& Barstow, M. A. 1993, ApJ, 416, L79

Gies, D. R. \& Wiggs, M. S. 1991, ApJ, 375, 321

Gruendl, R. A., Chu, Y.-H., O’Dwyer, I. J., \& Guerrero, M. A. 2001, AJ, 122, 308

Haffner, L. M. et al. 2003, ApJS, 149, 405

Hartmann, D., \& Burton, W. B. 1997, Atlas of Galactic Neutral Hydrogen, Cambridge University Press, ISBN 0521471117

Hewett, P. C., et al. 2003, ApJ, 599, L37

Holberg, J. B., Barstow, M. A., \& Sion, E. M. 1998, ApJS, 119, 207

Kruk, J. W., \& Werner, K. 1996, in H-Deficient Stars, eds. U. Heber \& C. S. Jeffery, the ASP Conference Series, 96, 205

Kruk, J. W., \& Werner, K. 1998, ApJ, 502, 858

O’Dwyer, I. J., Chu, Y.-H., Gruendl, R. A., Guerrero, M. A., \& Webbink, R. F. 2003, AJ, 125,2239

Otte, B., Dixon, W. V. D., \& Sankrit, R. 2004, ApJ, 606, L143

Parker, R. A. R., Gull, T. R., \& Kirshner, R. P. 1979, An Emission-Line Survey of the Milky Way, NASA SP-43A

Rauch, T., Kerber, F., \& Pauli, E.-M. 2004, A\&A, 417, 647

Reynolds, R. J. 1987, ApJ, 315, 234

Reynolds, R. J. 1988, ApJ, 333, 341

Sion, E. M. \& Downes, R. A. 1992, ApJ, 396, L79

Sion, E. M., Holberg, J. B., Barstow, M. A., \& Scheible, M. P. 1997, AJ, 113, 364

Walborn, N. R. 1973, AJ, 78, 1067

Werner, K., Bagschik, K., Rauch, T., \& Napiwotzki, R. 1997, A\&A, 327, 721 
Werner, K., Heber, U., \& Fleming, T. 1994, A\&A, 284, 907

Werner, K., et al. 1996, A\&A, 307, 860

Wilson, R. E. 1953, General Catalogue of Stellar Radial Velocities, Carnegie Institute Washington D.C. Publication, 601 


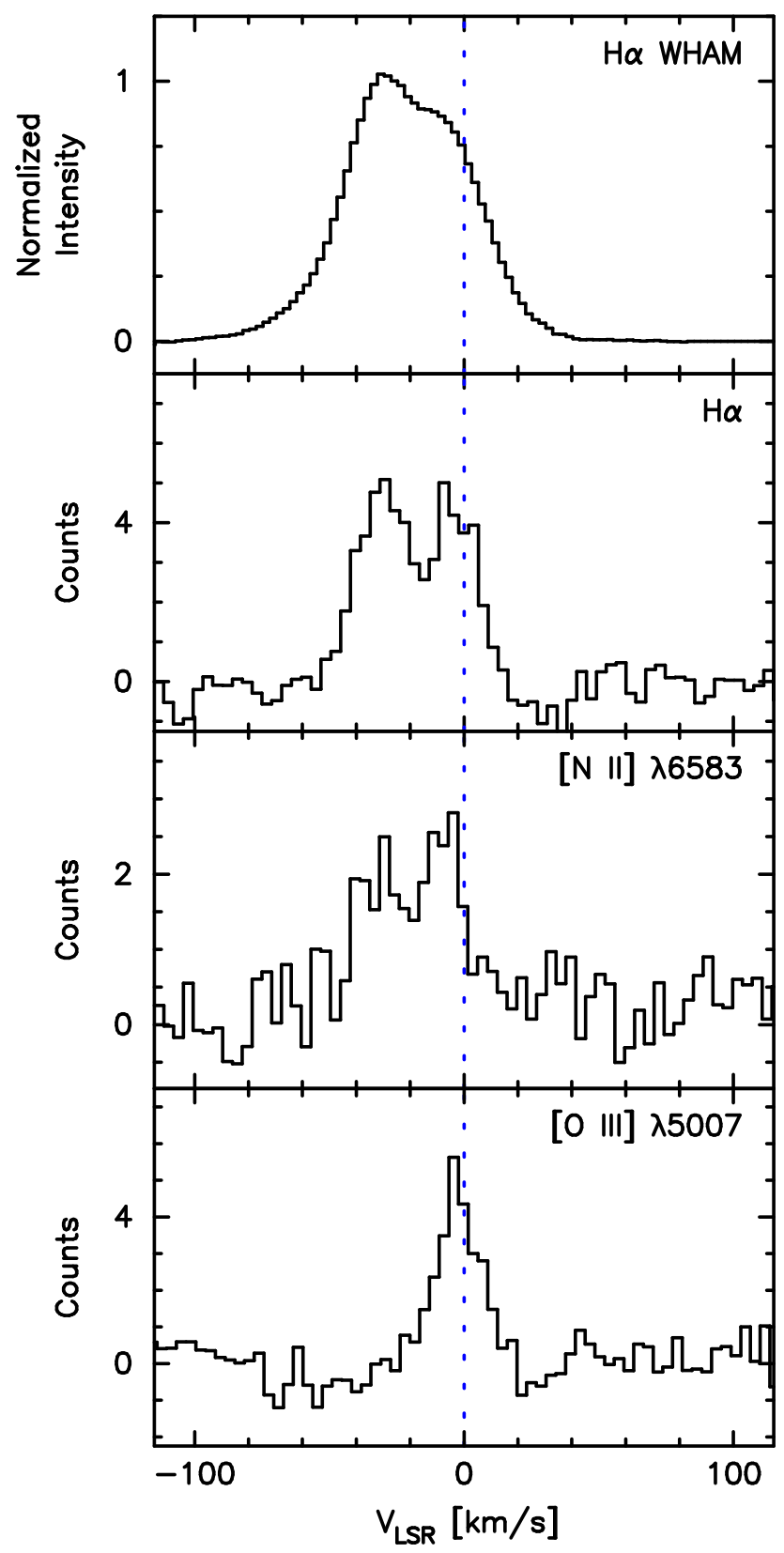

Fig. 1.- Velocity profiles of the $\mathrm{H} \alpha,[\mathrm{N} I \mathrm{II}]$, and [O III] lines. The $\mathrm{H} \alpha$ line profile in the top panel is extracted from the WHAM-NSS and normalized to its peak. The other line profiles are extracted from our echelle observations. 

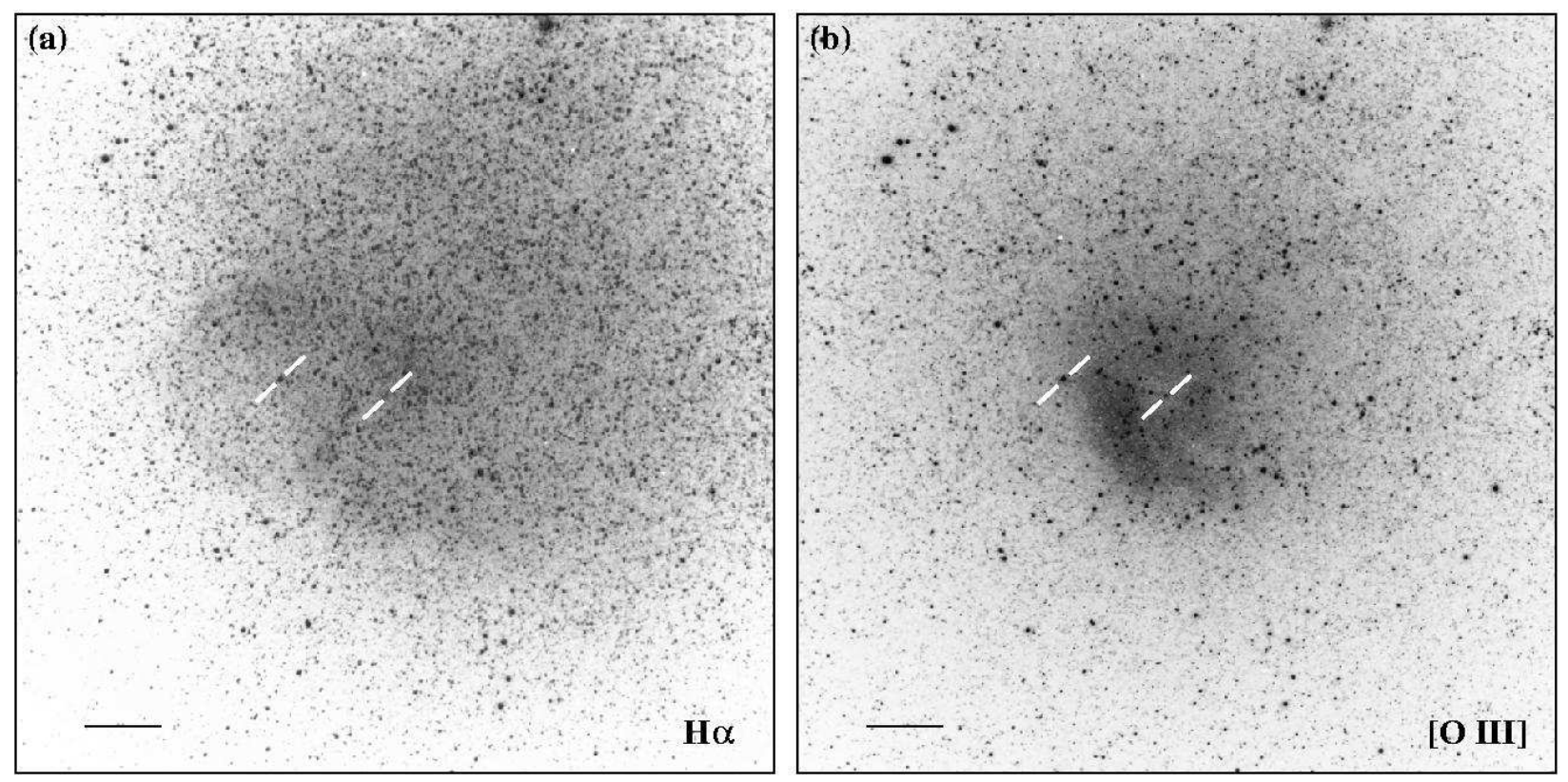

Fig. 2.- (a) H $\alpha$ and (b) [O III] images from Gull \& Chu emission-line survey of the northern sky, using a 135-mm-focal-length lens. North is up and east is to the left. The stars AO Cas (on the left) and KPD 0005+5106 (on the right) are marked. The [O III]-emission region is roughly centered at $\mathrm{KPD} 0005+5106$. The $1^{\circ}$ scale is marked by the horizontal line at the lower left corner of each panel. 

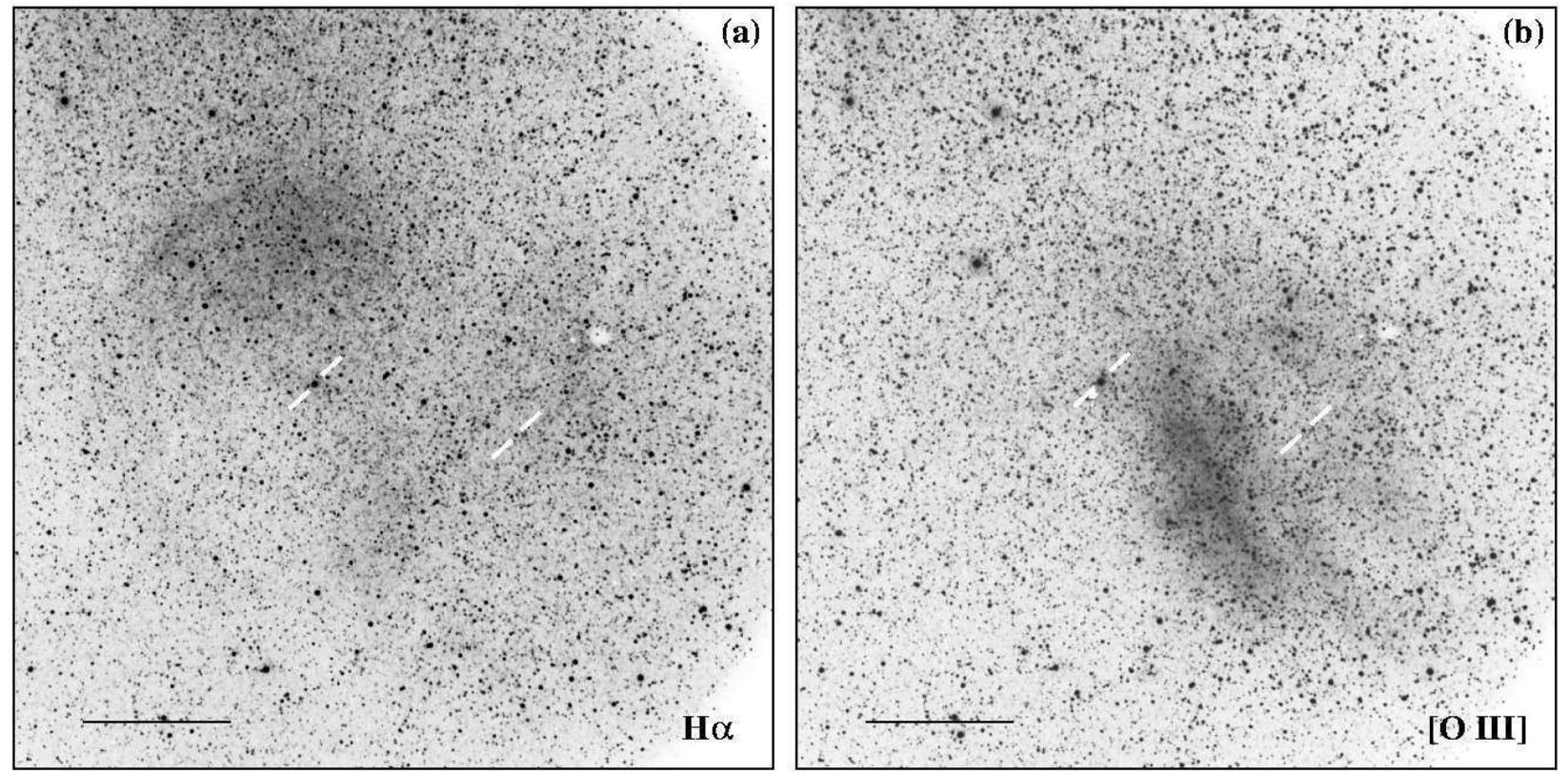

Fig. 3.- (a) $\mathrm{H} \alpha$ and (b) [O III] images from follow-up observations of An Emission-Line Survey of the Milky Way (Parker et al. 1979), using a 300-mm-focal-length lens. The [O III]emission region is roughly centered at KPD $0005+5106$. The $1^{\circ}$ scale is marked by the horizontal line at the lower left corner of each panel. 


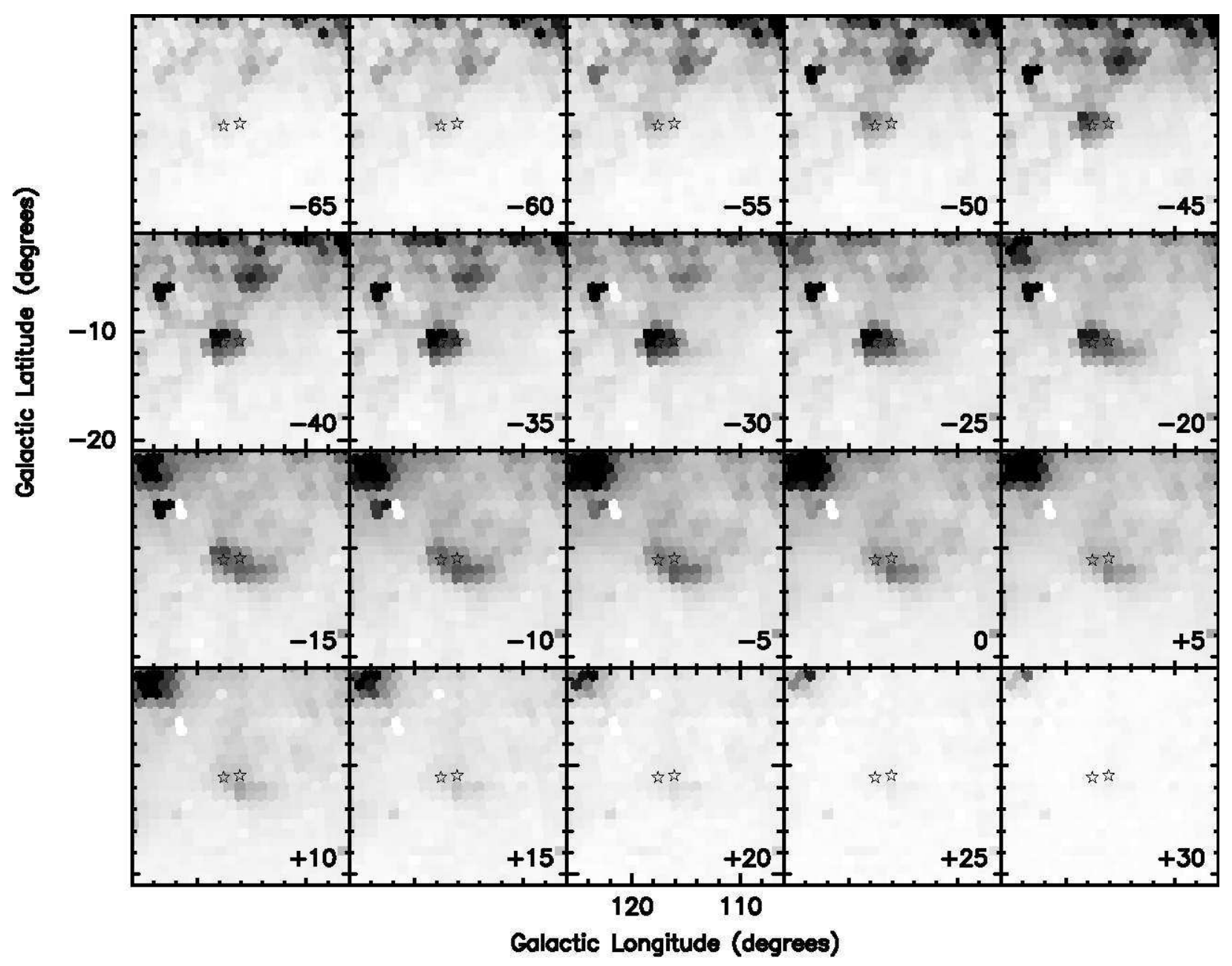

Fig. 4.- Iso-velocity maps extracted from the WHAM-NSS data. The local standard of rest velocity $\left(V_{\mathrm{LSR}}\right)$ of each panel is marked at the lower right corner. The two stars marked are AO Cas (on the left) and KPD 0005+5106 (on the right). These maps are presented in the galactic coordinates, so the orientation is rotated counterclockwise by about $15^{\circ}$ with respect to that of Figure 2. 